EPJ Web of Conferences 41, 05006 (2013)

DOI: $10.1051 /$ epjconf/20134105006

(C) Owned by the authors, published by EDP Sciences, 2013

\title{
Influence of the Chemical Design on the Coherent Photoisomerization of Biomimetic Molecular Switches
}

\author{
Jérémie Léonard $^{1}$, Dario Polli ${ }^{2}$, Giulio Cerullo ${ }^{2}$, Massimo Olivucci ${ }^{3,4}$, Stefan Haacke $^{1}$ \\ ${ }^{1}$ Institut de Physique et Chimie des Matériaux de Strasbourg, CNRS - Université de Strasbourg, \\ France \\ ${ }^{2}$ IFN-CNR, Dipartimento di Fisica, Politecnico di Milano, Piazza L. da Vinci, 32, 20133 Milano, \\ Italy \\ ${ }^{3}$ Chemistry Department, Bowling Green State University, Bowling Green, United States \\ ${ }^{4}$ Dipartimento di Chimica, Università degli Studi di Siena, Italy \\ e-mail: Jeremie.Leonard@ipcms.unistra.fr
}

\begin{abstract}
Ultrafast transient absorption spectroscopy reveals the effect of chemical substitutions on the photoreaction kinetics of biomimetic photoswitches displaying coherent dynamics. Ground state vibrational coherences are no longer observed when the excited state lifetime exceeds $300 \mathrm{fs}$.
\end{abstract}

\section{INTRODUCTION}

Photoisomerizing molecular switches convert light energy into mechanical energy at the molecular scale. Naturally implemented in photosensitive proteins to trigger their biological activity, such molecules may also be artificially synthesized to control a given functionality at the molecular level, or to drive molecular machines with light. To this end, the indanylidene-pyrroline (IP) chemical skeleton has been designed to reproduce the excited state $\left(\mathrm{S}_{1}\right)$ potential energy surface of retinal in rhodopsin (Rho) [1, 2]. As a result, these biomimetic molecules undergo an ultrafast photoisomerization involving coherent dynamics through a conical intersection (CI) [3]. This is a remarkable biomimetic process in which a significant amount of mechanical energy is delivered into a few specific vibrational modes. The IP molecular family appears as a powerful model system, where combined transient absorption spectroscopy and state-of-the-art modeling (QM/MM) allows a detailed, mechanistic understanding of the coherent dynamics [4].

Several representatives of the IP molecules are now available with various substitutions (see Fig. 1A). Here we investigate the influence of chemical substitutions on the photoreaction dynamics. We perform transient absorption spectroscopy on two new, cationic members of the family, the NAIP and $\mathrm{dMe}-\mathrm{MeO}-\mathrm{NAIP}$, which are respectively missing the $\mathrm{MeO}$ group on $\mathrm{C}_{6}$, or the $\mathrm{Me}$ group on $\mathrm{C}_{5}$. We compare the dynamics to that of ZW-MeO-NAIP [3], itself very similar to MeO-NAIP [4]. In the VIS range, the successive signatures of Stimulated Emission (SE) and Photoproduct Absorption (PA) may be observed, allowing us to pinpoint accurately in time the passage of the system through the CI, as was also observed in Rho [5]. We show that unlike the photoreaction of NAIP which is the fastest and also vibrationally coherent, that of dMe-MeO-NAIP is significantly slower and no longer displays coherent dynamics.

This is an Open Access article distributed under the terms of the Creative Commons Attribution License 2.0, which permits unrestricted use, distribution, and reproduction in any medium, provided the original work is properly cited. 


\section{Results}

For the previously reported IPs the fluorescence is shorter-lived than the Excited State Absorption (ESA) observed in UV transient absorption experiments [2-4]. A more accurate measurement of the residence time in $S_{1}$ resides in the crossover between SE and PA. It is observed in several of the IPS displaying vibrationally coherent dynamics, and also in the case of NAIP as shown in Fig. 1B and 1C. This data is obtained with a $400-\mathrm{nm}, 60$-fs pump and a supercontinuum VIS probe with the setup described in $[2,3]$. At time zero, SE is observed at $\lambda>470 \mathrm{~nm}$, an intense ESA band is seen at $\lambda<$ $470 \mathrm{~nm}$ and a weaker, very short-lived one at $\lambda>550 \mathrm{~nm}$. While time evolves, the red ESA $(>550 \mathrm{~nm})$ rapidly vanishes and reveals that the SE extends very far towards long wavelengths $(>$ $650 \mathrm{~nm})$, where it is suddenly followed by the PA signal. The latter rapidly blue shifts to form the longer-lived band at $\lambda<470 \mathrm{~nm}$. This observation of far red-detuned, successive signals of SE and PA is the signature of a population, which is relatively well focused in the configuration space in form of a vibrational wavepacket, and evolves through the CI. The vibrational coherence is further illustrated by the oscillations seen in the PA signature in the 430 -nm trace at times $>250 \mathrm{fs}$ (fig. 1C), as already reported for other IPs [3, 4].

(A)

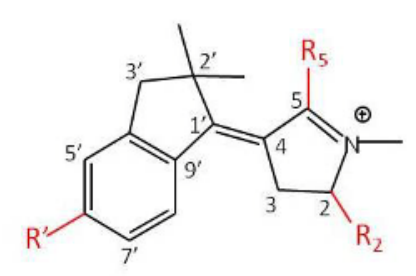

(B)

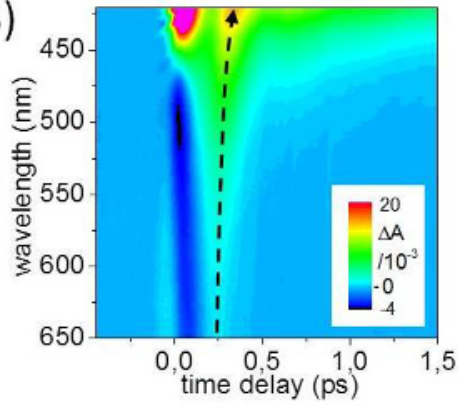

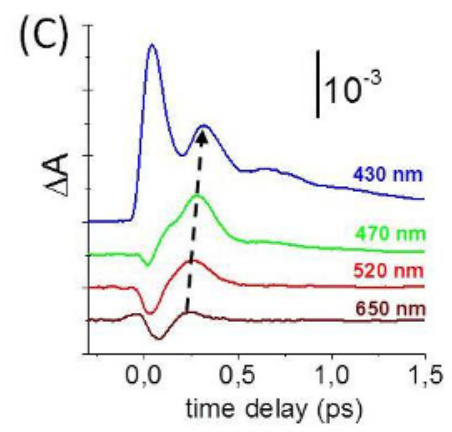

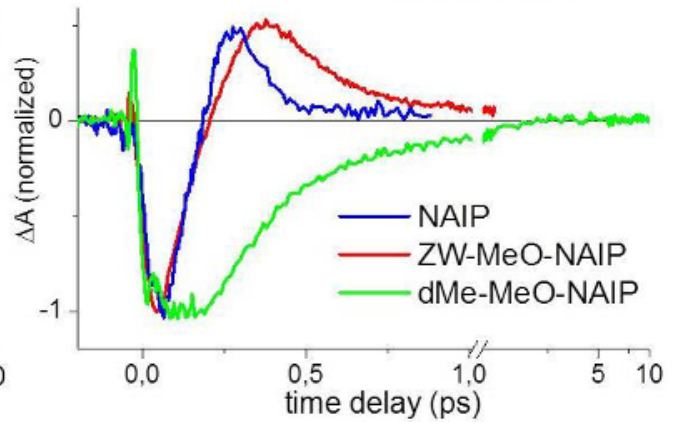

(E)

(D)

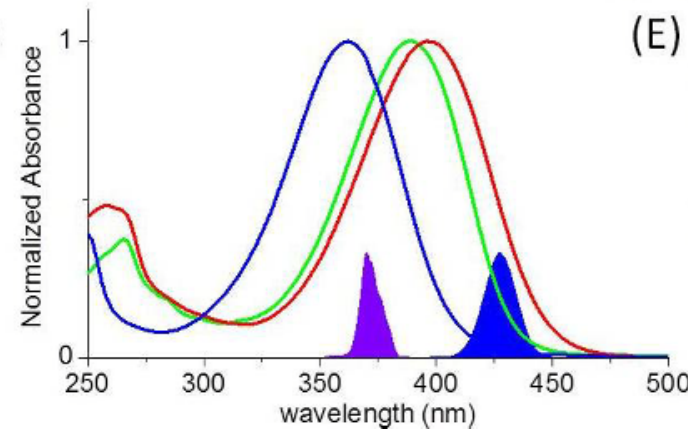


frequency generation of the 780-nm fundamental pulse with a VIS or IR NOPA [6] in order to pump the NAIP compound at $370 \mathrm{~nm}$ or the other two compounds at $425 \mathrm{~nm}$. Fig 1E displays the kinetic traces observed at $520 \mathrm{~nm}$ for the 3 compounds. In the case of ZW-MeO-NAIP, the maximum of the positive PA contribution is observed after $380 \mathrm{fs}$, in perfect agreement with previous results [3]. The NAIP compound displays a similar kinetic trace, but a faster decay to $\mathrm{S}_{0}$, with the PA signal maximum at $280 \mathrm{fs}$. Instead, the dMe-MeO-NAIP shows a qualitatively different kinetic trace, with no signature of the PA at $520 \mathrm{~nm}$, unlike in all other IP compounds. The constant signal level up to $\sim 200$ fs may simply be due to the overlapping wing of the same, weak, red ESA band as seen in other IPs. Then, the SE signal decays with a $\sim 0.3$-ps time constant. Furthermore, while NAIP displays signatures of coherent dynamics through the CI like other IPs, dMe-MeO-NAIP does not [7].

\section{Conclusion}

The photoreaction speed and coherent dynamics of the IP photoswitches can be tuned by chemical substitutions. The impulsive appearance of the PA signal in the VIS region is a signature of vibrational coherence, which is preserved in NAIP, where the $\mathrm{MeO}$ group is removed and the photoreaction is faster, presumably due to an enhanced slope in the $S_{1}$ potential energy surface towards the CI. Instead no such signature is observed in dMe-MeO-NAIP, where the longer $\mathrm{S}_{1}$ lifetime exceeds the typical vibrational coherence lifetime in the IPs [3, 4]. Presumably, the nonplanar $\mathrm{S}_{0}$ geometry of ZW-MeO-NAIP, imposed by the steric hindrance of the Me group at $\mathrm{C}_{5}$, accelerates the photoreaction as compared to the quasi-planar dMe-MeO-NAIP. Remarkably, there seems to be no correlation between the reaction speed or the appearance of vibrational coherence in the photoproduct, and the photoisomerization quantum yield. A detailed investigation addressing this point is in progress.

\section{Acknowledgement}

We acknowledge support from the European Community (FP-7 INFRASTRUCTURES-2008-1, "Laserlab Europe II", Contract No. 228334).

\section{References}

1. Lumento, F., et al., Quantum Chemical Modeling and Preparation of a Biomimetic Photochemical Switch. Angew. Chem. Int. Ed. 46: p. 414-420 (2007).

2. Sinicropi, A., et al., An artificial molecular switch that mimics the visual pigment and completes its photocycle in picoseconds. Proc. Nat. Acad. Sc. USA. 105: p. 17642-17647 (2008).

3. Briand, J., et al., Coherent Ultrafast Torsional Motion and Isomerisation of a Biomimetic Dipolar Photoswitch. PCCP. 12: p. 3178-3187 (2010).

4. Léonard, J., et al., Mechanistic Origin of the Vibrational Coherence Accompanying the Photoreaction of Biomimetic Molecular Switches. submitted, (2012).

5. Polli, D., et al., Conical intersection dynamics of the primary photoisomerization event in vision. Nature. 467(7314): p. 440 (2010).

6. Manzoni, C., D. Polli, and G. Cerullo, Two-color pump-probe system broadly tunable over the visible and the near infrared with sub-30 fs temporal resolution. Rev Sci Instrum. 77(2): p. 023103-9 (2006).

7. Dunkelberger, A.D., et al., Photoisomerization and Relaxation Dynamics of a Structurally Modified Biomimetic Photoswitch. J. Phys. Chem. A. 116: p. 3527-3533 (2012). 